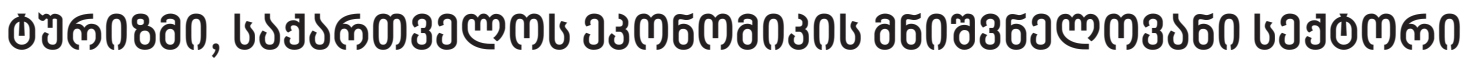

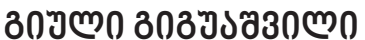

https://doi.org/10.35945/gb.2017.04.020

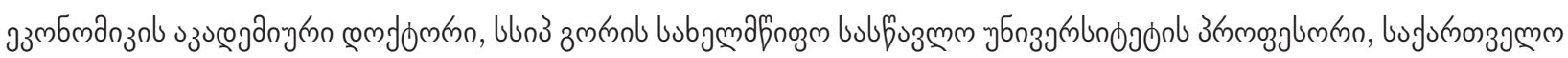

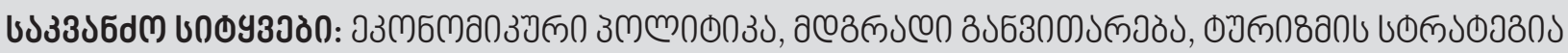

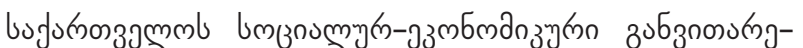

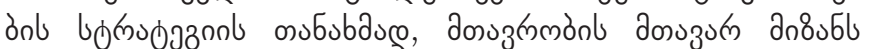

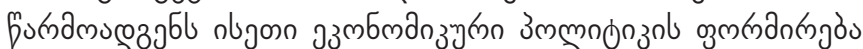

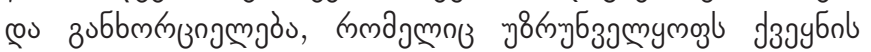

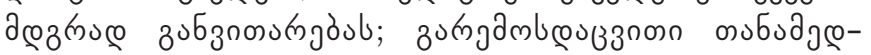

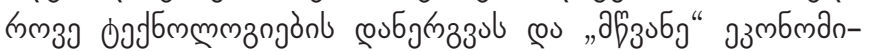

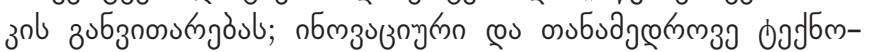

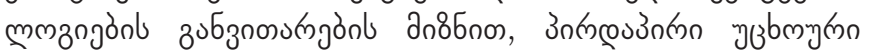

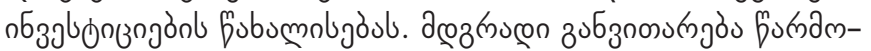

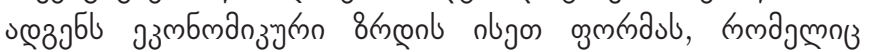

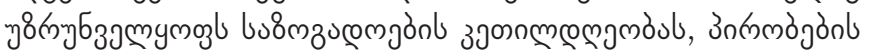

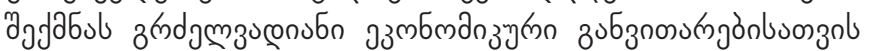

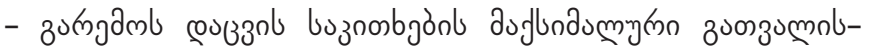

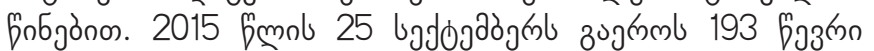

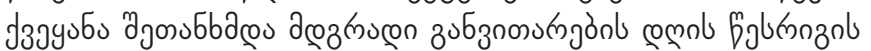

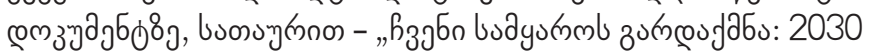

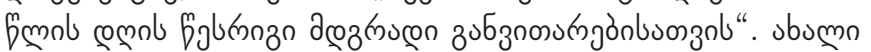

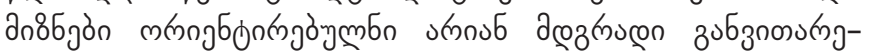

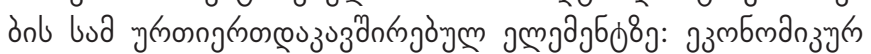

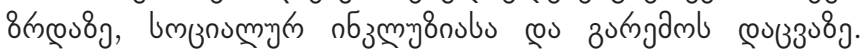

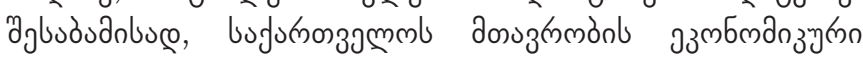

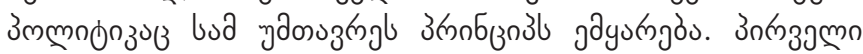

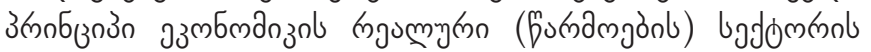

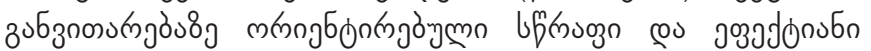

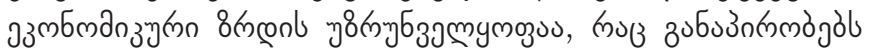

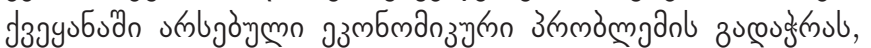

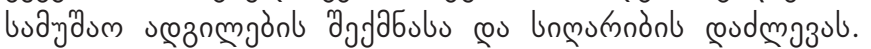

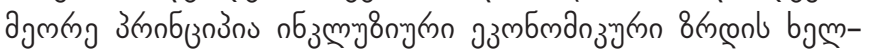

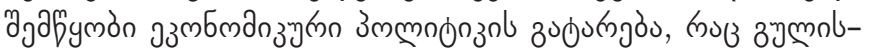

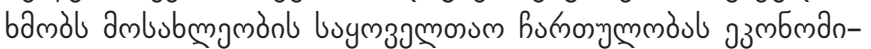

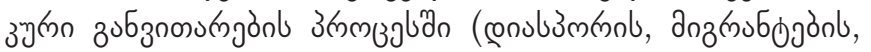

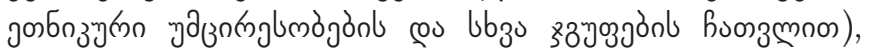

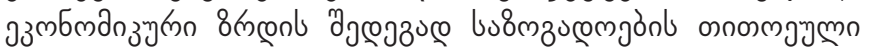

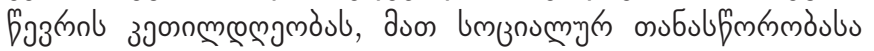

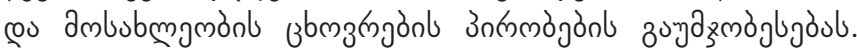

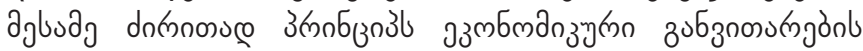

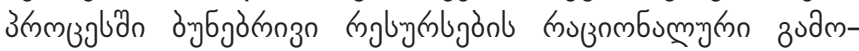

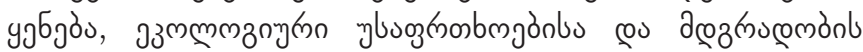

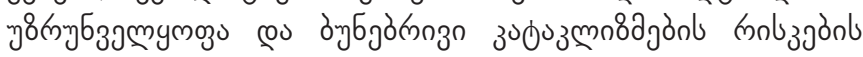

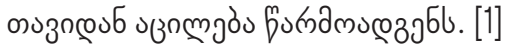

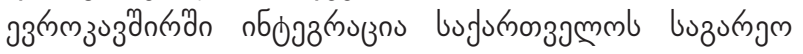

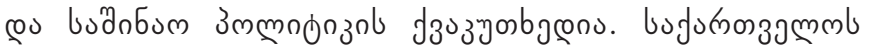

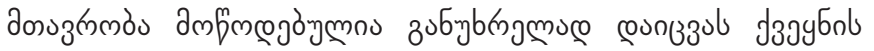

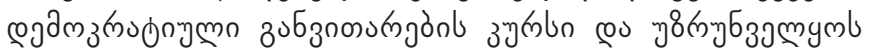

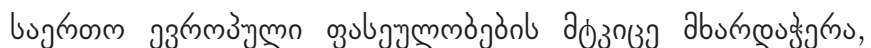

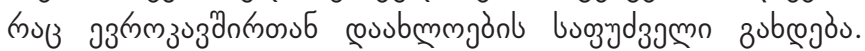

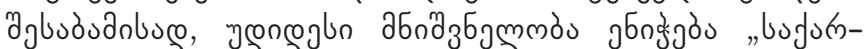

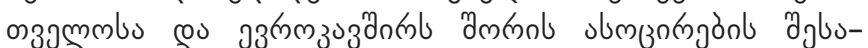
bjo "g gos

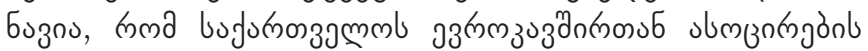

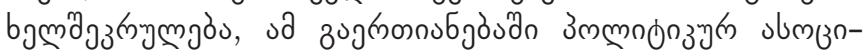

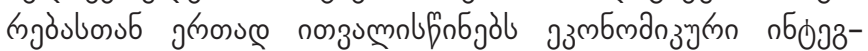

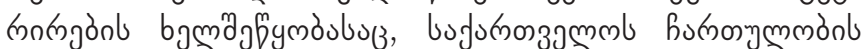

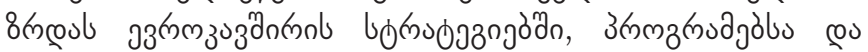

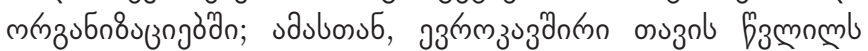

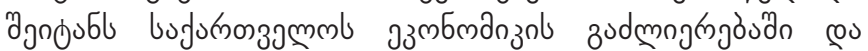

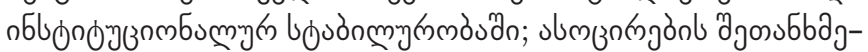

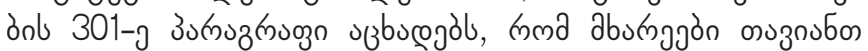

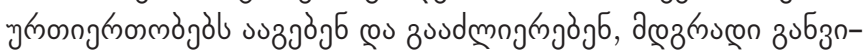

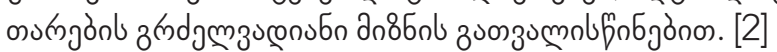

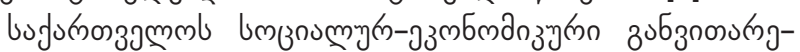

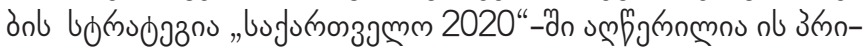

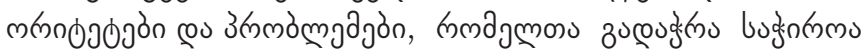

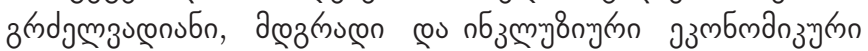

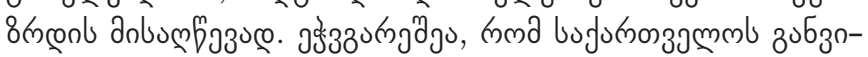

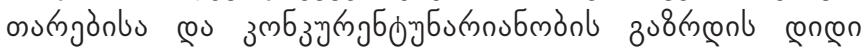

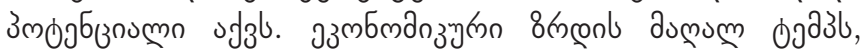

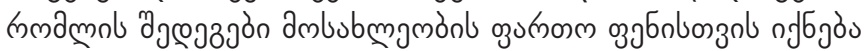

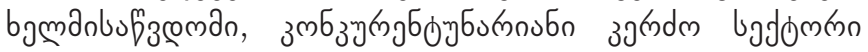

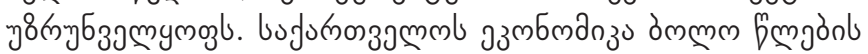

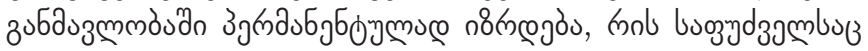

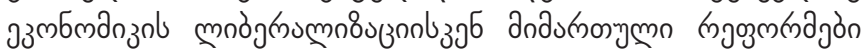

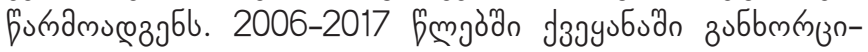

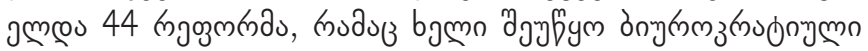

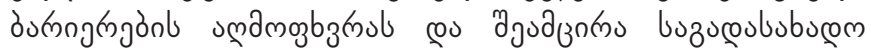
o3n rरon.

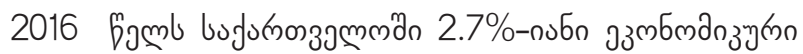

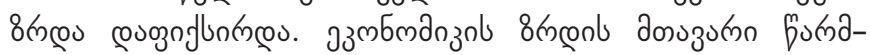

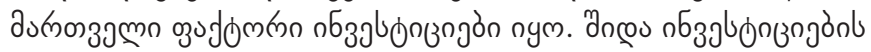

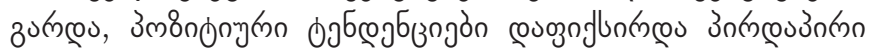

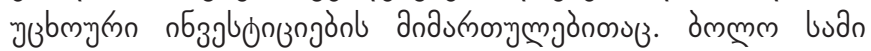

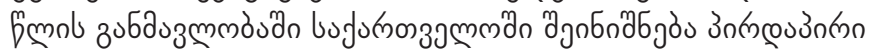

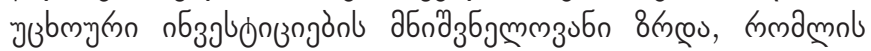

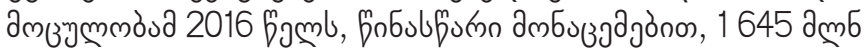

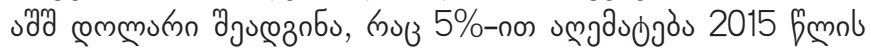
ambusjajall. [3]

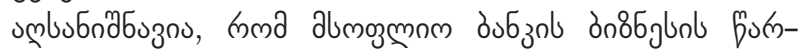

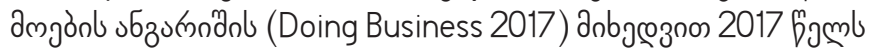




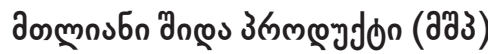

\begin{tabular}{|c|c|c|c|c|c|c|}
\hline & 2011 & 2012 & 2013 & 2014 & 2015 & 2016 \\
\hline 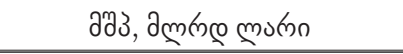 & 24.3 & 26.2 & 26.8 & 29.2 & 31.8 & 33.9 \\
\hline 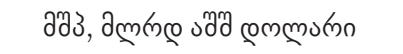 & 14.4 & 15.8 & 16.1 & 16.5 & 14.0 & 14.3 \\
\hline 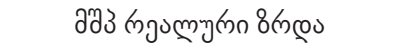 & $7.2 \%$ & $6.4 \%$ & $3.4 \%$ & $4.6 \%$ & $2.9 \%$ & $2.7 \%$ \\
\hline 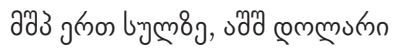 & 3,231 & 3,523 & 3,600 & 3,676 & 3,767 & 3,852 \\
\hline
\end{tabular}

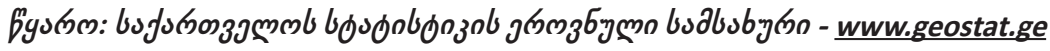

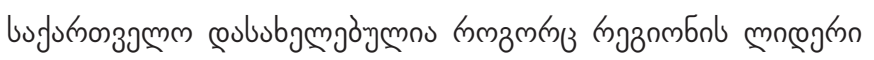

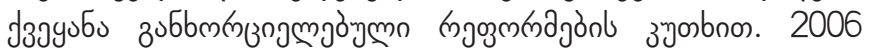

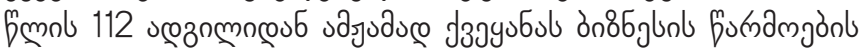

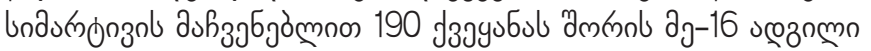
эз०3००.

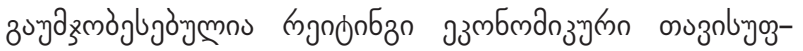
mgònl nbegflunl (The Heritage Foundation 2017) anbjęn-

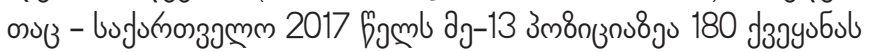

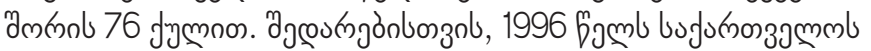

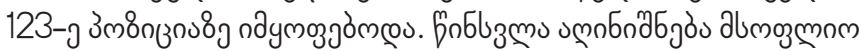

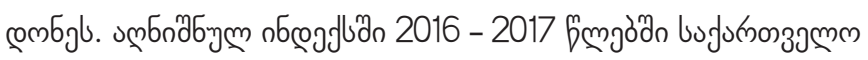

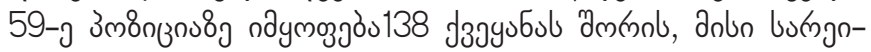

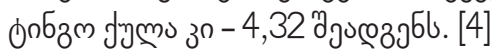

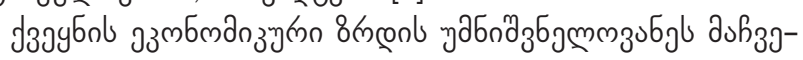

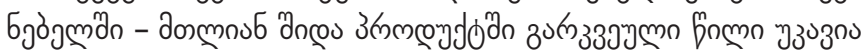

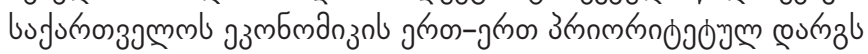

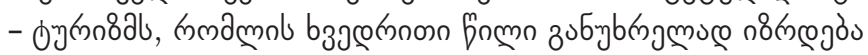

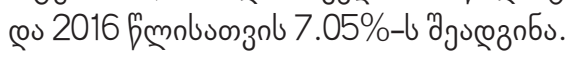

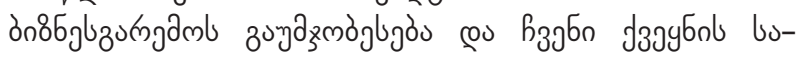

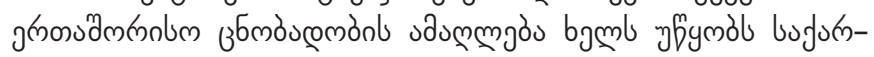

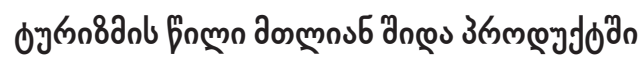

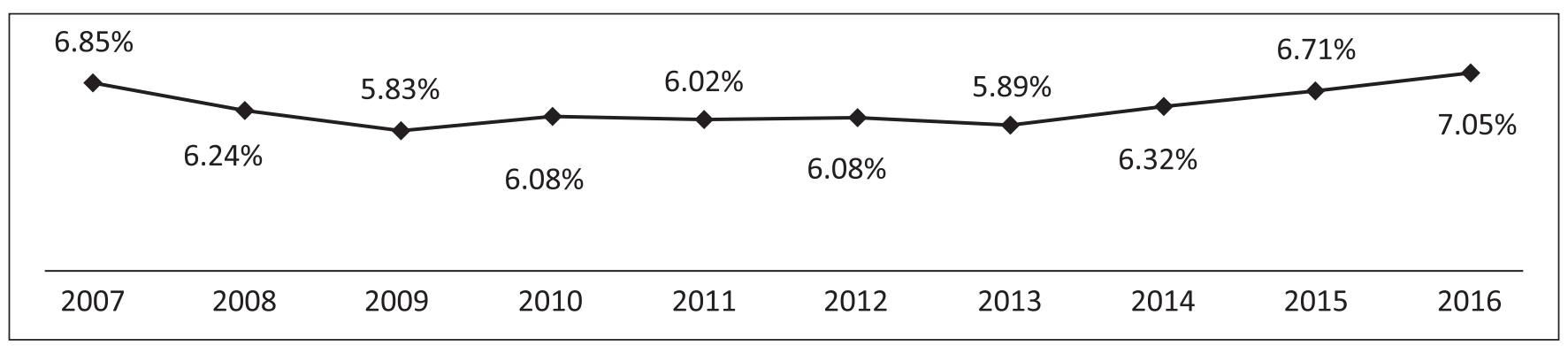

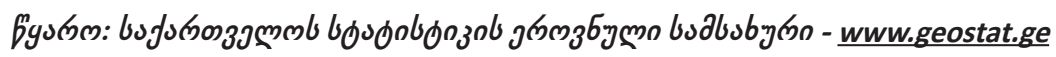

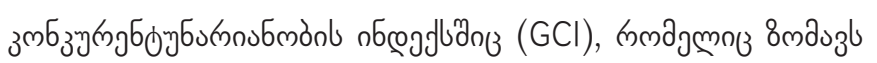

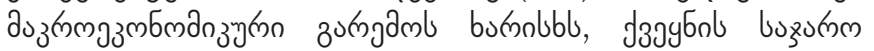

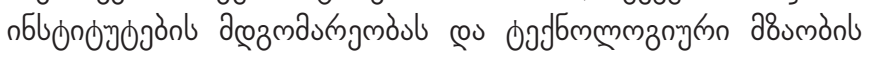

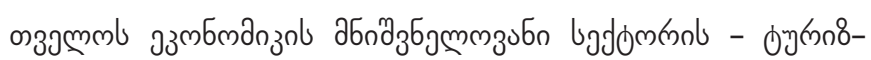

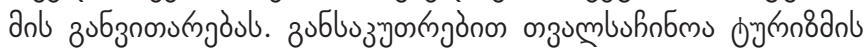

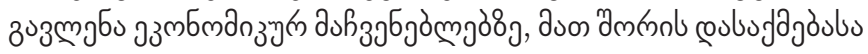

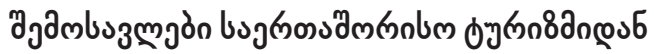

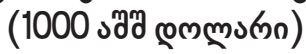

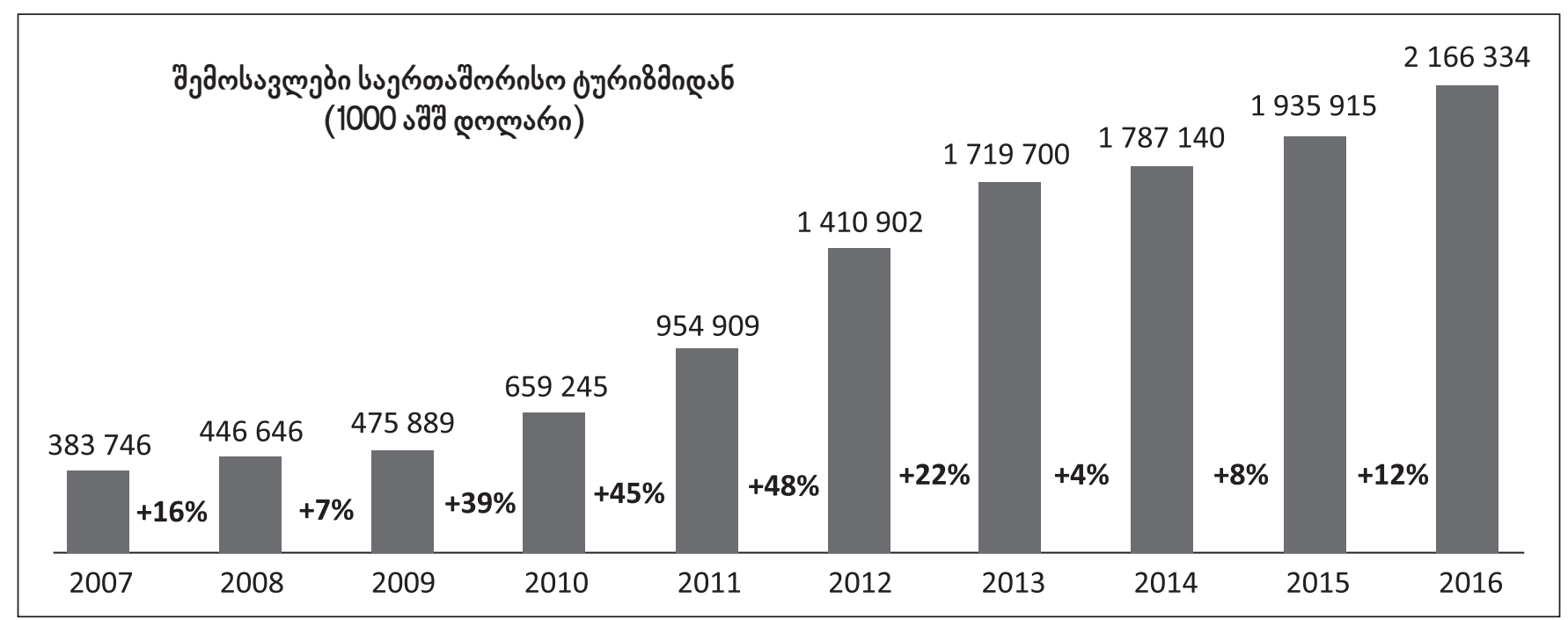

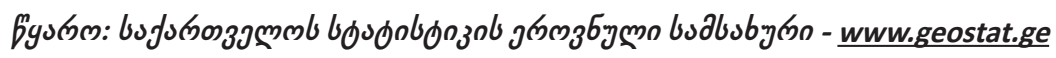




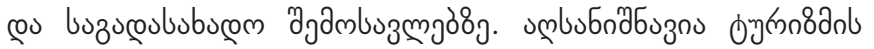

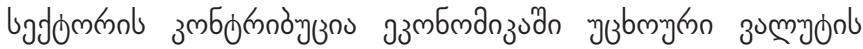

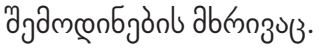

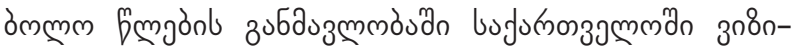

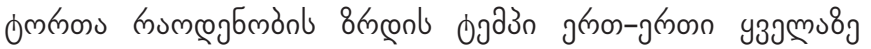

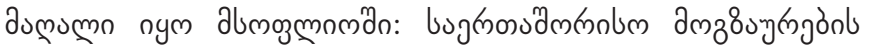

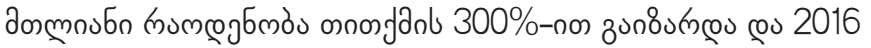

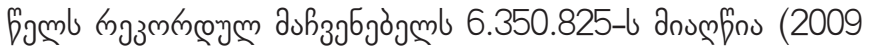

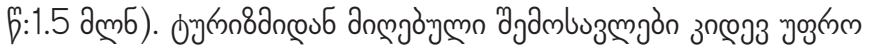

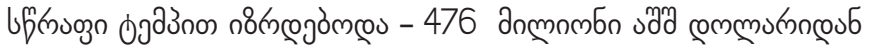

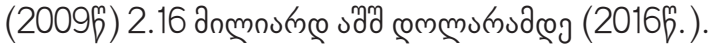

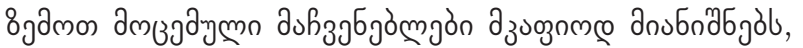

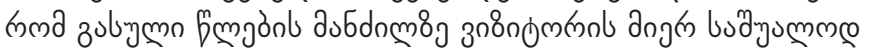

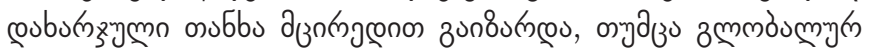

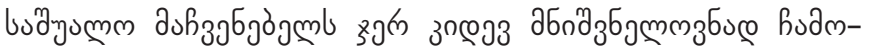

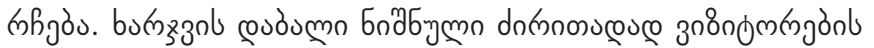

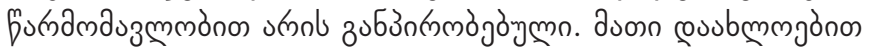

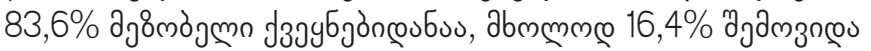

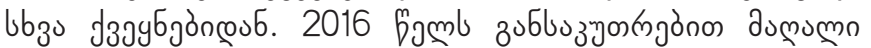

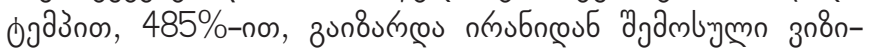

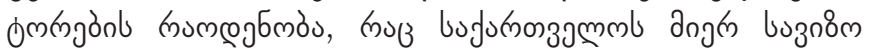

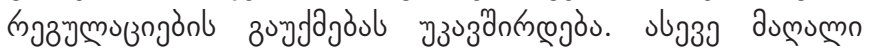

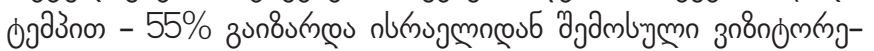

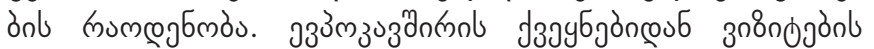

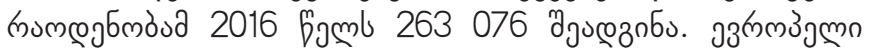

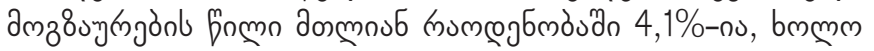

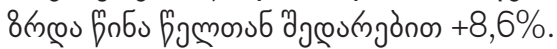

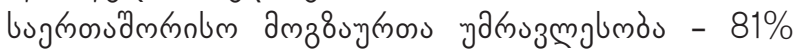

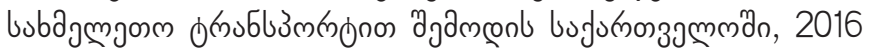

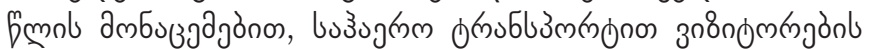

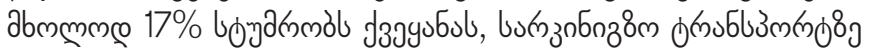

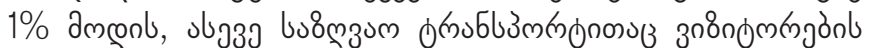

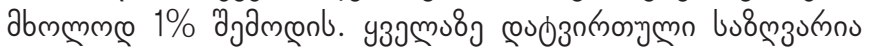

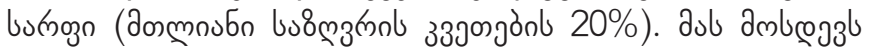
18\%-no buøubmm қu fromgmo bnen 18 \%-non.

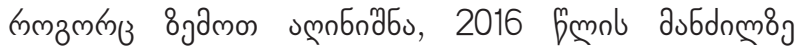

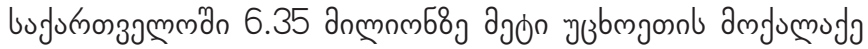

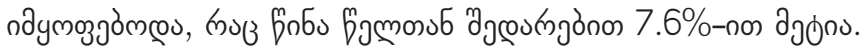

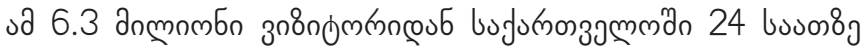

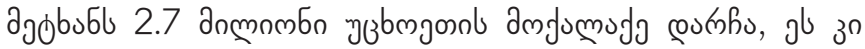

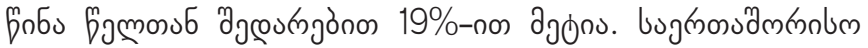

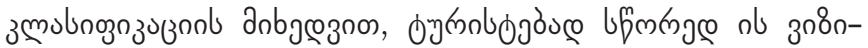

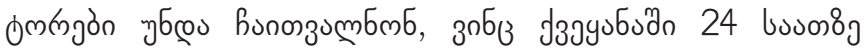

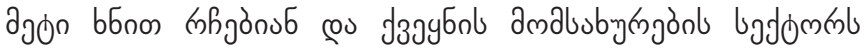

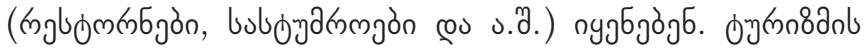

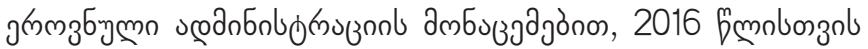

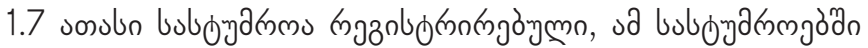

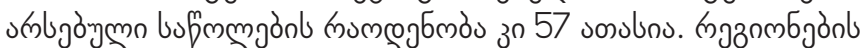

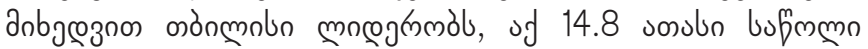

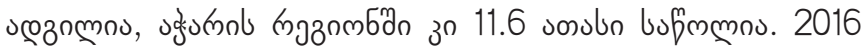

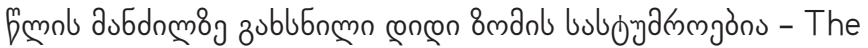
Biltmore Tbilisi, Crowne Plaza, Gino Wellness cou bbzaòn.

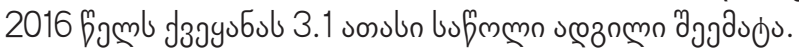

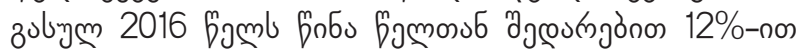

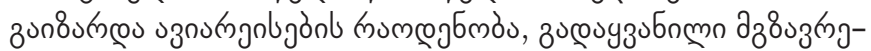

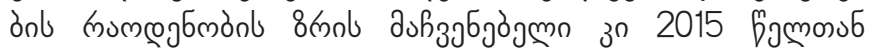

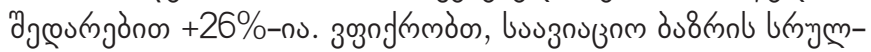

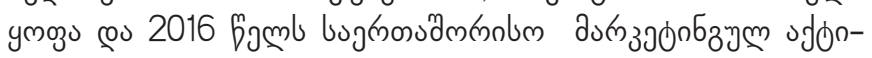

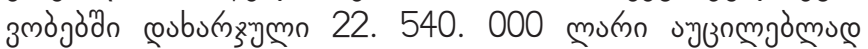

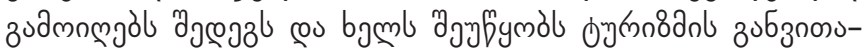

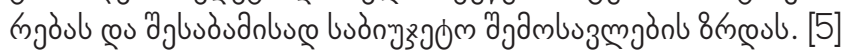

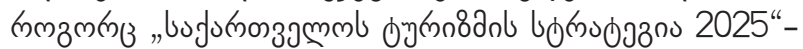
Ins zublus

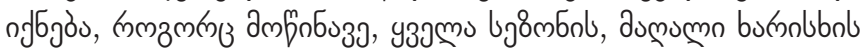

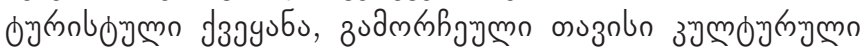

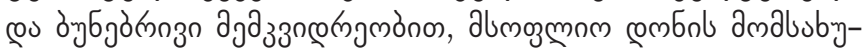

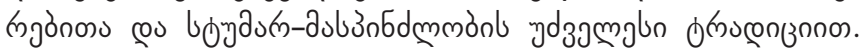

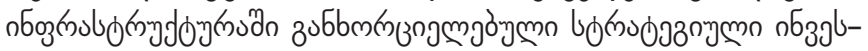

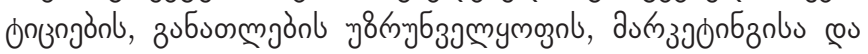

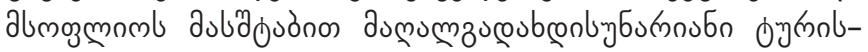

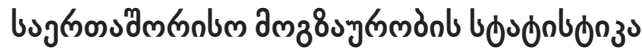

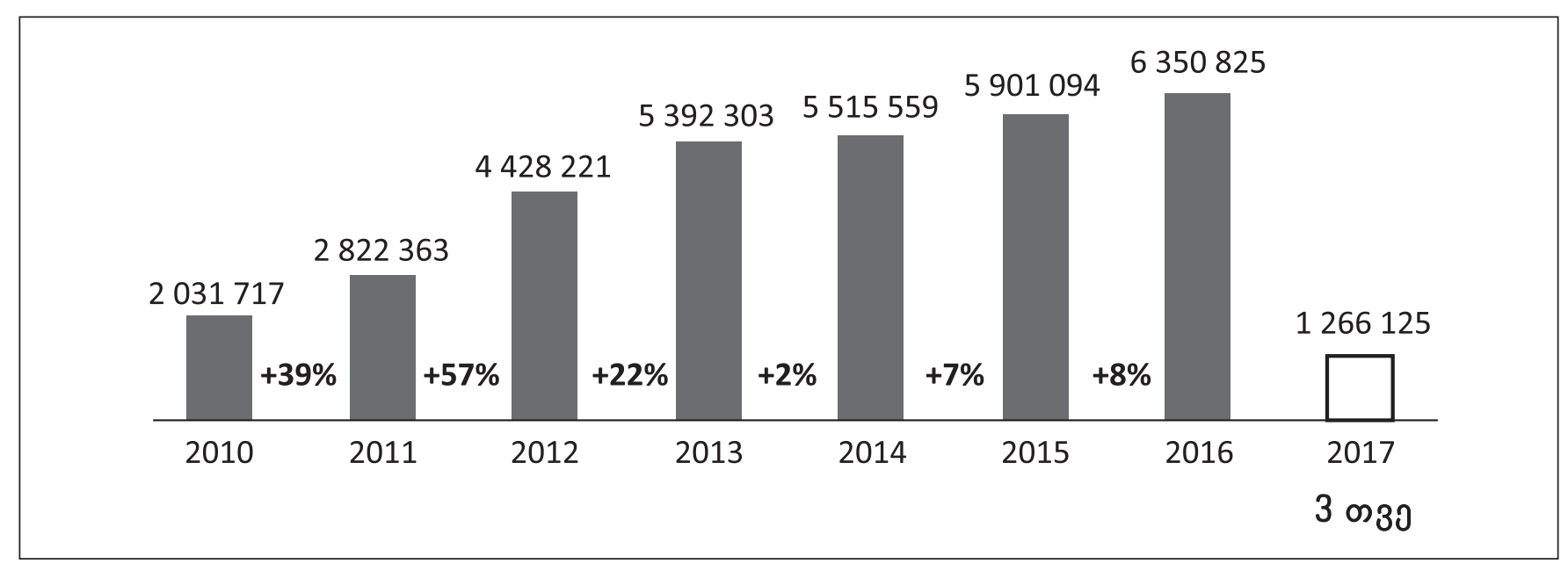

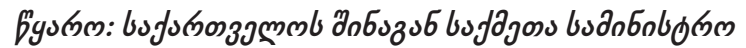




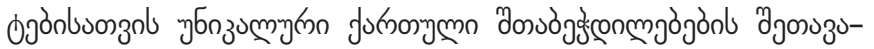

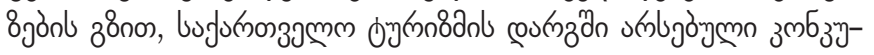

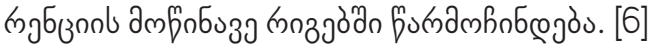

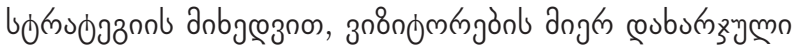

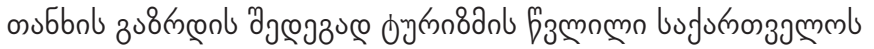

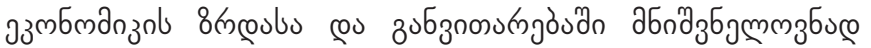

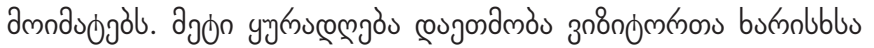

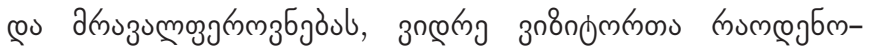

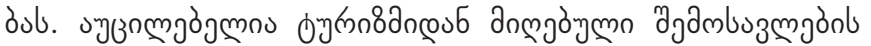

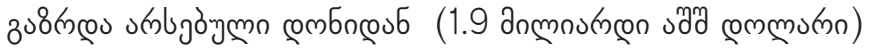

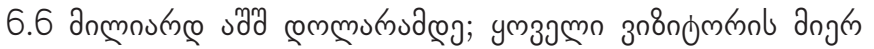

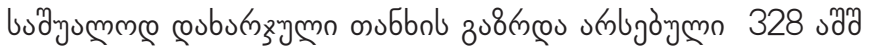

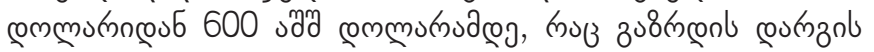

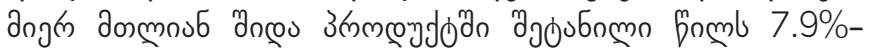

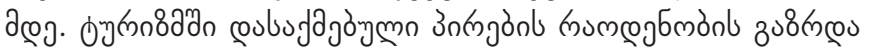

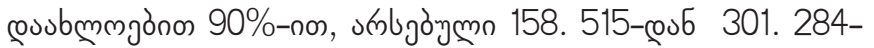

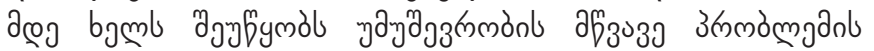

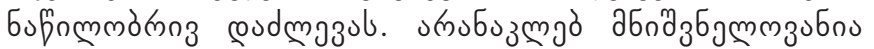

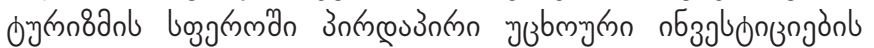

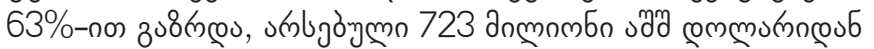

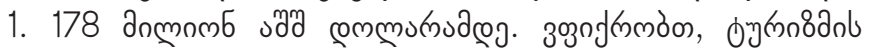

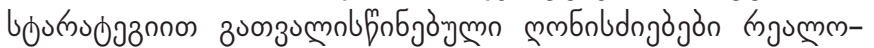

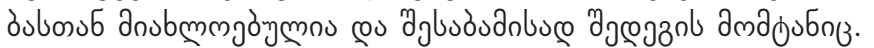

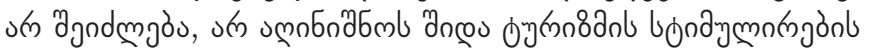

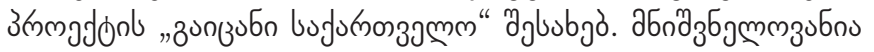

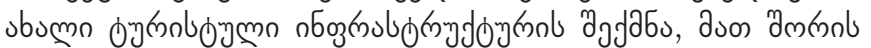

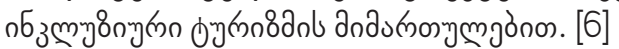

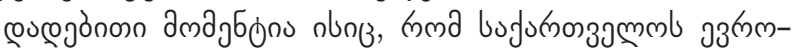

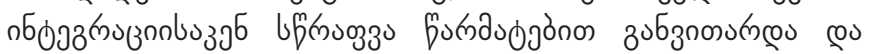

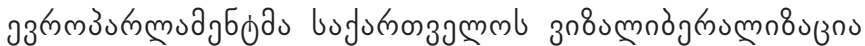

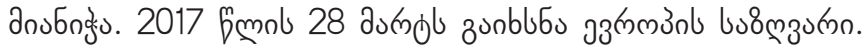

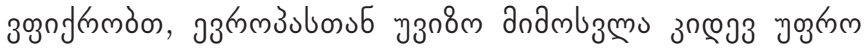

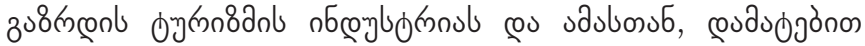

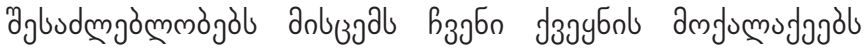

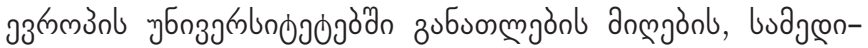

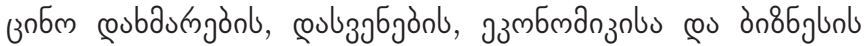
zubznonsmänls उचonbon.

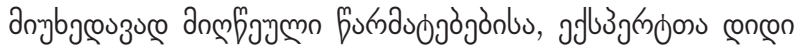

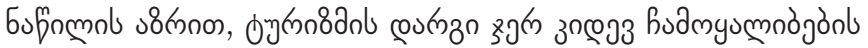

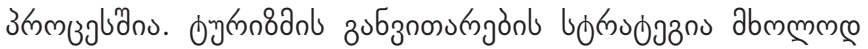

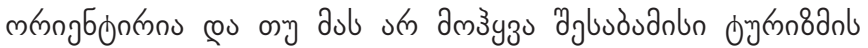

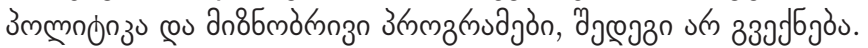

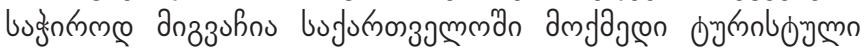

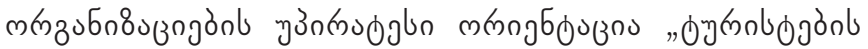

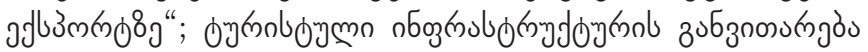

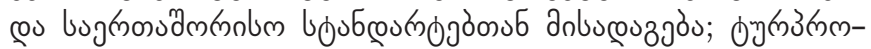

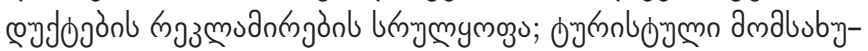

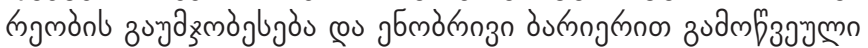

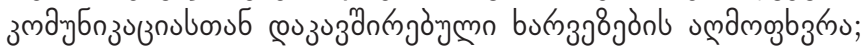

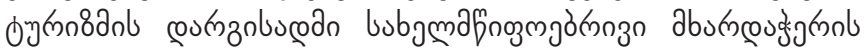

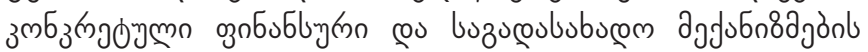

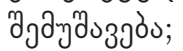

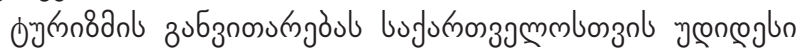

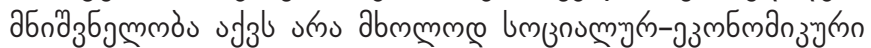

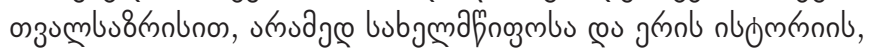

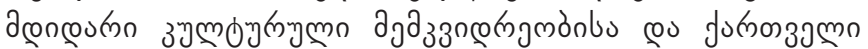

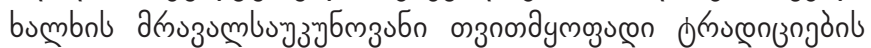

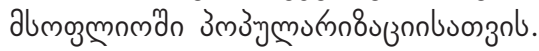

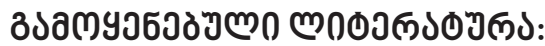

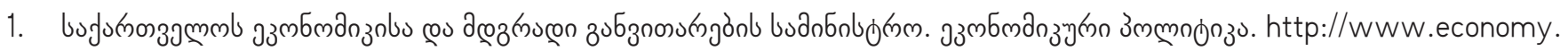
ge/?page =ecopolitic\&s=43

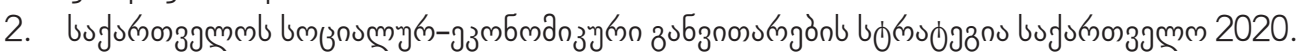

3. http://www.economy.ge/uploads/ecopolitic/2020/saqartvelo_2020.pdf

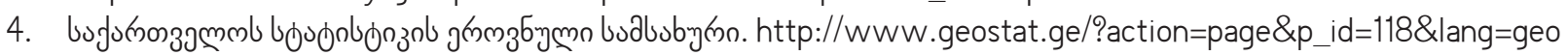

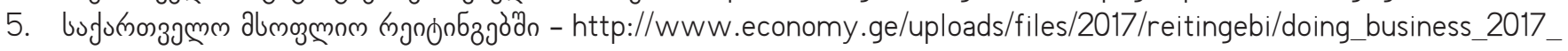
geo.pdf

6. bufunoszamml ongno

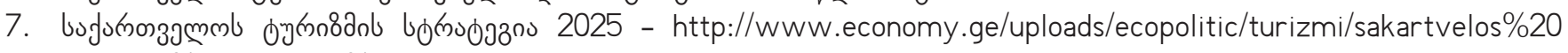
turizmis\%20strategia\%202025.pdf 


\title{
TOURISM AN IMPORTANT SECTOR OF GEORGIA'S ECONOMY
}

\author{
GIULI GIGUASHVILI
}

https://doi.org/10.35945/gb.2017.04.020

Academic Doctor of Economics, Professor

Gori State Teaching University, Georgia

\section{KEYWORDS: ECONOMIC POLICY, SUSTAINABLE DEVELOPMENT, TOURISM STRATEGY}

\section{SUMMARY}

Turism is considered as the largest and fastest growing industry in the World. Tourism's impact on the economic and social development of a country can be enormous: opening it up for business, trade and capital investment, creating jobs and entrepreneurialism for the workforce and protecting heritage and cultural values.

This sphere can bring much profit as to private firms, also to concrete countries, Revenues from tourism sector grow on annual basis and reach record figure. Over the past 10 years, tourism is an only sector in Georgian economy that grows on annual basis. It should be also noted that tourism sector has become one of the important sources for currency inflows to the country. According to the statistics of national tourism administration, the year of 2016 and the beginning of 2017 were especially successful in terms tourist and currency inflows from tourism sector.

Tourism in Georgia is an important component of the economy of Georgia. International tourism is a rapidly growing industry. In 2015 it employed around 158,500 people, producing $6.7 \%$ of Georgia's GDP by providing US \$1.94 billion of revenue. 2015 's tourists numbers reached a record high of 2,278,562 people. In 2016, international tourist arrivals increased again, reaching $2,714,773$, with resultant revenue estimated to exceed US \$2 billion.
Revealed 6,350,825 international visitors arrived in Georgia last year, which is 449,731 more than the year previous. The largest number of tourists is reported to come from Azerbaijan (1,523,075, an increase of 9.3\%) Second place is Armenia $(1,496,246$, an increase of $1.9 \%)$ followed by Turkey $(1,254,089$ - a decreased of $9.9 \%$ from last year). In the fourth place was Russia with 1,037,564 tourists visiting Georgia in 2016, a full $12 \%$ more than in 2015 . Visits from Ukraine increased by $21.8 \%$, amounting to 172,631 people. The latest data indicate year-on-year visitors from Europe also show positive signs; with the total number of travellers from Lithuania, Latvia, Czech Republic, Bulgaria and Germany all significantly increasing. An exceptional increase in tourism was observed from the following countries: Iran (+485\%), India (+ $199 \%)$, Saudi Arabia (+116\%), the Philippines (+ $89 \%)$, Oman (+75\%), and China (+46\%)," Chogovadze said.

The newly-established visa-free travel represents the most important achievement for Georgian citizens that will further deepen and encourage contacts, business relations and tourism.

Development of turistical business and involve in the economical service of the country is the most important and active problem of the development of market economics. For its regulating and arranging the state must do the best. 